\title{
Pattern of cerebral palsy seen in children attending the outpatient paediatric physiotherapy clinics in Osun State tertiary hospitals in Nigeria
}

\author{
J O Omole, ${ }^{1}$ B Physio, MSc (PT); S A Adegoke, ${ }^{2,3} \mathrm{MB}$ ChB, FWACP, MPH, PhD; K O Omole, ${ }^{3}$ MBBS, FWACP; \\ O A Adeyemi, ${ }^{4}$ BSc, MSc (Public Health) \\ ${ }^{1}$ Department of Physiotherapy, Obafemi Awolowo University Teaching Hospitals Complex, Ile-Ife, Osun State, Nigeria \\ ${ }^{2}$ Department of Paediatrics and Child Health, Obafemi Awolowo University, Ile-Ife, Osun State, Nigeria \\ ${ }^{3}$ Department of Paediatrics, Obafemi Awolowo University Teaching Hospitals Complex, Wesley Guild Unit, Ilesha, Osun State, Nigeria \\ ${ }^{4}$ Department of Physiotherapy, Ladoke Akintola University Teaching Hospital, Osogbo, Osun State, Nigeria
}

Corresponding author: J O Omole (elidrwiz@yahoo.com)

\begin{abstract}
Background. Cerebral palsy (CP) is a major cause of disability in children and the most commonly encountered neurologic condition by paediatric physiotherapists in Nigeria. Local data on the pattern of presentation of CP and standardised management protocols are lacking. Objectives. To assess the pattern of CP seen in children attending paediatric physiotherapy clinics in Osun State tertiary hospitals.

Methods. A hospital-based cross-sectional study was conducted in three tertiary hospitals within Osun State, Nigeria. Data were collected using caregiver questionnaires, medical records and physical assessment (Gross Motor Function Measure-88) and were recorded on a standardised case record form. Data were analysed using appropriate statistical tests with alpha set at $p<0.05$.

Results. A total of 187 children with CP were seen during the six-month period. The male to female ratio was 1.2:1 and the children were aged 12 months to 12 years. The majority of the mothers (63.6\%) were primiparous and, at the time of delivery, most mothers were aged between 28 and 33 years. Spastic (72.7\%) and quadriplegic (69.5\%) presentations were the leading sub-types of CP, with $76.5 \%$ of children having one or more associated problems. Birth asphyxia (57.2\%) was the leading aetiology of CP while speech impairment was common in $63.6 \%$ of cases. One hundred and fourteen (61\%) children were classified as being severely disabled and $53.5 \%$ had a gross motor function measure score of less than $40.9 \%$.

Conclusion. Severe CP is commonly encountered in the region, with children most frequently presenting with spastic quadriplegia and speech impairment. A good knowledge of the pattern of CP seen in south-western Nigeria is one of the first steps in developing a standardised protocol.
\end{abstract}

S Afr J Child Health 2018;12(2):52-57. DOI:10.7196/SAJCH.2018.v12i2.1452

Neurological disorders are common in childhood, with cerebral palsy (CP) being one of the leading causes of disability ${ }^{[1,2]} \mathrm{CP}$ is a disorder of abnormal posture with scarcity of movement caused by lesions in an immature or developing brain with varying degrees of associated problems including seizure disorders, intellectual disabilities, communication difficulties, learning difficulties, visual impairment, bladder and bowel control problems and swallowing difficulties. ${ }^{[3]}$ The prevalence of $\mathrm{CP}$ in well-resourced countries is between 1.5 to 2.5 per 1000 live births while in Africa the prevalence is between 1.5 and 10 per 1000 live births. ${ }^{[4-8]}$ Reasons for this disparity have been attributed to poor government policies on healthcare, harmful traditional beliefs, higher rates of unsupervised deliveries and inadequate equipment to implement resuscitative procedure following complicated labour in many African countries. ${ }^{[9]}$

$\mathrm{CP}$ can occur during the prenatal, perinatal or postnatal stages. ${ }^{[10]}$ In some cases, the aetiology of $\mathrm{CP}$ is not known, however, some common identifiable causes include birth asphyxia, severe jaundice/ kernicterus, infections, neonatal seizures, prematurity and low birth weight. ${ }^{[5,10]}$ Diagnosis of CP is made by clinical evaluation with or without cerebral imaging. Failure to identify aetiology in a child with a neurological condition does not exclude CP, provided the brain injury that resulted in the motor function deficits occurred before the child was older than three years of age. ${ }^{[10]}$ Children with $\mathrm{CP}$ require lifelong healthcare, by a range of professional disciplines (including paediatrician, neurologist, orthopaedic surgeon, physiotherapist, occupational therapist and speech therapist), using substantial human and financial resources. ${ }^{[11,12]}$
Physiotherapy plays a major role in the management of children with $\mathrm{CP}^{\left[{ }^{[13]}\right.}$ The aims of physiotherapy intervention include: strengthening of the mother-to-child bonding, optimisation of functional skills, physical endurance and motor development, therein facilitating school participation via provision of mobility devices, advising and facilitating appropriate handling and positioning and preventing complications such as contractures and other deformities. Physiotherapy treatment approaches include neurodevelopmental therapy, sensory integration therapy, conductive education, constraint induced movement therapy, context focused therapy, advance neuromotor rehabilitation, biofeedback and physical activity training. ${ }^{[13,14]}$ Despite the important rehabilitative role of physiotherapy in the management of children with CP in Nigeria, standardised protocols or guidelines are lacking. Therefore, the aim of this study was to describe the pattern of CP in children attending the paediatric physiotherapy clinics located in tertiary hospitals, Osun State, Nigeria, to inform the development of a standardised clinical guideline for the physiotherapy management of the children.

\section{Methods}

\section{Study design and participants}

This was a hospital-based, cross-sectional, descriptive study of consecutive children attending the paediatric physiotherapy clinics of three tertiary hospitals in Osun State, Nigeria, over a six-month period. There are also primary and secondary healthcare facilities available in the state but the physiotherapy services are inadequate at secondary centres. Children were eligible for inclusion if they were 
aged between 12 months and 12 years, and had a documented referral from a paediatrician or neurologist confirming the diagnosis of $\mathrm{CP}$. Children with other neurological conditions such as spina bifida, Down's syndrome, and poliomyelitis were excluded from the study.

\section{Procedure}

Ethical approval (ref. no. ERC/2017/06/34) was obtained from the Ethics and Research Committee of the Obafemi Awolowo University Teaching Hospitals Complex, Ile-Ife, Nigeria. Approval was also obtained from the heads of the Departments of Physiotherapy and Paediatrics at the study sites. Informed consent for participation of the children in the study was obtained from the parents/caregivers prior to enrolment. Parents/caregivers accompanying the children were asked to fill in a questionnaire. Information obtained included present age (mother, child, and primary caregiver if not the mother), sex, the birth order of the child with CP in the family, level of education of the primary caregiver and their spouse, occupation of the primary caregiver and their spouse, and monthly income.
Information obtained from medical records and physical assessment included: aetiology; classification of CP based on the topographical distribution (diplegia, hemiplegia and quadriplegia); nature of the movement disorder (spastic, dyskinetic, ataxic, mixed and hypotonic); severity of $\mathrm{CP}$ using the Gross Motor Function Classification System - Expanded and Explained (GMFCS-ER); the extent of motor impairments using the Gross Motor Function Measure-88 (GMFM-88); and associated problems such as intellectual disabilities, seizure disorders, and speech and hearing impairments.

The GMFCS-ER is a reliable and valid tool for assessment of the severity of $\mathrm{CP}^{[15]}$ The tool focuses on sitting, transfer and mobility ,which are all self-initiated movements. It assesses five levels of function, with Level I indicating that ambulation is possible with no restriction, while Level $\mathrm{V}$ indicates that self-ambulation is not possible and mobility is only achievable using a wheelchair. Five age categories are used in this scale: $<2$ years, $2-4$ years, $4-6$ years, $6-12$ years and $12-18$ years. ${ }^{[15]}$

\section{Table 1. General characteristics of children with cerebral palsy and their mothers $(N=187)$}

\begin{tabular}{|c|c|}
\hline Variables & $n(\%)^{*}$ \\
\hline Age of children (years), median (IQR) & $3.0(1.0-5.0)$ \\
\hline Mother's age at child's birth (years), median (IQR) & $28.0(24.0-33.0)$ \\
\hline Parents'/caregivers' age (years) during study, median (IQR) & $34.0(28.0-39.0)$ \\
\hline \multicolumn{2}{|l|}{ Children's sex } \\
\hline Male & $103(55.1)$ \\
\hline Female & $84(44.9)$ \\
\hline \multicolumn{2}{|l|}{ Children's age at first contact during study (years) } \\
\hline $1-2$ & $48(25.7)$ \\
\hline $2-4$ & $44(23.5)$ \\
\hline $4-6$ & $52(27.8)$ \\
\hline $6-12$ & $43(23.0)$ \\
\hline \multicolumn{2}{|l|}{ Children's birth order } \\
\hline 1 st & $119(63.6)$ \\
\hline 2nd & $37(19.8)$ \\
\hline $3 \mathrm{rd}$ & $15(8.0)$ \\
\hline 4 th & $7(3.8)$ \\
\hline$\geq 5$ th & $9(4.8)$ \\
\hline \multicolumn{2}{|l|}{ Age range of mothers (at child's birth) of children with CP (years) } \\
\hline $17-21$ & $10(5.3)$ \\
\hline $22-27$ & $67(35.8)$ \\
\hline $28-33$ & $78(41.7)$ \\
\hline $34-39$ & $23(12.3)$ \\
\hline$\geq 40$ & $9(4.8)$ \\
\hline \multicolumn{2}{|l|}{ Socioeconomic status } \\
\hline Upper class & $44(23.5)$ \\
\hline Middle class & $61(32.6)$ \\
\hline Lower class & $82(43.9)$ \\
\hline \multicolumn{2}{|l|}{ Educational background } \\
\hline Primary & $15(8)$ \\
\hline Secondary & $71(38)$ \\
\hline Post secondary & $101(54)$ \\
\hline \multicolumn{2}{|l|}{ Monthly income in Nigerian Naira (\#) } \\
\hline$<18000$ & $52(27.8)$ \\
\hline $18000-50000$ & $66(35.3)$ \\
\hline $51000-100000$ & $32(17.1)$ \\
\hline $101000-150000$ & $24(12.8)$ \\
\hline$>150000$ & $13(7)$ \\
\hline
\end{tabular}


Table 2. Aetiology of cerebral palsy and associated problems $(N=187)$

\begin{tabular}{ll}
\hline Aetiology, $n$ (\%) & $107(57.2)$ \\
Birth asphyxia & $32(17.1)$ \\
CNS infection & $22(11.8)$ \\
Kernicterus & $3(1.6)$ \\
Neonatal seizure & $14(7.5)$ \\
Premature birth & $3(1.6)$ \\
Trauma & $6(3.2)$ \\
Unknown & \\
Associated problems, $n(\%)$ & $119(63.6)$ \\
Speech impairment & $22(11.8)$ \\
Bladder control problems & $25(13.4)$ \\
OMI & $9(4.8)$ \\
Intellectual disability & $10(5.3)$ \\
Learning disability & $40(21.4)$ \\
Seizure disorders & $4(2.1)$ \\
Emotional and behavioural disorder & $11(5.9)$ \\
Visual impairment & $4(2.1)$ \\
Hearing impairment & $44(23.5)$ \\
None &
\end{tabular}

The GMFM - 88 is also a reliable and valid tool consisting of 88 domains sub-divided into the following 5 items: Item A - Lying and Rolling, Item B - Sitting, Item C - Crawling and Kneeling, Item D - Standing and Item E - Walking, Running and Jumping. Scoring ranges from 0 ('does not initiate') to 3 ('completes'). ${ }^{[16]}$ The advantage of this scale is that it allows professionals to objectively assess motor performance changes over time. ${ }^{[17]}$

\section{Data analysis}

Socioeconomic status of parents of children with CP was calculated using the Ogunlesi et al.$^{[18]}$ classification of social class. The method is a modification of an earlier classification done by Oyedeji. ${ }^{[19]}$ The previous classification did not take into account the parents' income to assign socioeconomic scores - hence the need for this modification. In summary, socioeconomic scores were allotted to both educational qualification and occupation based on the equivalents of each parent's mean income using their percentile incomes. ${ }^{[18]}$ Socioeconomic class was scored as $1,2,3,4$ and 5, with the social class represented as I, II, III IV and V, respectively. Socioeconomic class was further sub-classified as: $(i)$ a total score of 1 or 2 representing social classes I and II were sub-classified as upper class; (ii) a total score of 3 representing social class III was sub-classified as middle class; (iii) a total score of 4 or 5 representing social classes IV and V were sub-classified as lower class.

Continuous variables such as age were summarised using median and interquartile range (IQR), while categorical variables such as sex, motor type, and topography were summarised using percentages and proportions. Associations between categorical variables were determined using the $\chi^{2}$ test. Data were analysed using Statistical Programme for Social Sciences (SPSS) for Windows version 22.0 (IBM Corp., USA). The alpha level was set at 0.05 .

\section{Results}

A total of 187 children with CP were included during the six months' study period. The median (IQR) age of the children was 3.0 (1.0 - 5.0) years with a male to female ratio of 1.2:1 while the median (IQR) age of mothers (at child's birth) was $28.0(24.0-33.0)$ years. General characteristics of children with $\mathrm{CP}$ and their mothers/caregivers was captured (Table 1). First-born children constituted the highest prevalence of CP cases seen in this study (63.6\%), while mothers in the age range of 28 - 33 years (41.7\%) gave the highest frequency. The parental/caregivers' socioeconomic status showed that upper class families constituted $23.5 \%$, while lower class families were 43.9\%. Post-secondary education of parents/caregivers constituted the highest prevalence seen (54\%) in this study, while monthly income between $\$ 18000$ and $\$ 50000$ was $35.3 \%$. The three most common individual aetiology were: birth asphyxia (57.2\%), central nervous system infection (18.2\%) and kernicterus (11.7\%) whilst the three most associated problems seen in this study were: speech impairments (63.6\%), seizure disorders $(21.4 \%)$ and oral motor impairment (including problems with feeding, swallowing and drooling) (13.4\%) (Table 2).

Distribution of children's severity levels, motor type, topography, age categories, and number of associated problems per child were also captured (Table 3). Spastic CP was the most common motor type (72.7\%) with severe deficits (GMFCS Levels IV and V) occurring in $73.5 \%$ of this group. Quadriplegic CP was the most common topographical distribution (69.5\%), accompanied by severe deficits in $79.2 \%$ of cases. Mild deficits (GMFCS Levels I and II) were generally associated with one or no co-morbidities; while more severe $\mathrm{CP}$ tended to present with more than one associated problems $\chi^{2}=53.22$ and $p=0.001$. A breakdown of the type of associated problems was conducted (Table 4). Speech impairment was the most prevalent single associated problem (25.7\%) whilst a combination of the three associated problems of speech impairment, visual impairment and seizure disorder $(3.7 \%)$ were the most prevalent. The pattern and relationship between gross motor function classification system and the gross motor function measure was analysed (Table 5). Mild deficits (Level I and II) of CP were associated with a lesser degree of motor impairment $(80-100 \%)$ while severe deficits of CP related to a higher degree of motor impairment $(0-20.9 \%)\left(\chi^{2}=291.82 ; p=0.002\right)$.

The gross motor function classification system was also significantly associated with other variables, i.e. socioeconomic status $\left(\chi^{2}=22.42\right.$; $p=0.004)$; topography $\left(\chi^{2}=144.26 ; p=0.001\right)$; motor type $\left(\chi^{2}=74.79\right.$; $p=0.001)$; and birth order $\left(\chi^{2}=55.77 ; p=0.001\right)$ (Table 6).

\section{Discussion}

This study showed a slight male bias of 1.2:1, in line with previous studies. ${ }^{[20-22]}$ Reasons for this bias may be attributed to male susceptibility to genetic mutations and variants in recessive X-linked chromosomes. ${ }^{[23]}$ A greater proportion of children who were firstborns (63.6\%) presented with CP in this study. Reasons for this may be due to the nature of healthcare delivery in Nigeria. Cultural preference and financial resource limitations may affect the choices of mothers in terms of home v. institutional delivery, and that the facilities and expertise available amongst different institutions are highly variable. ${ }^{[9]}$ Primigravid women are more at risk of prolonged obstructed labour, which may lead to birth asphyxia in babies and subsequent development of $\mathrm{CP}^{\left[{ }^{[24]}\right.}$ Hashim et al. ${ }^{[25]}$ concluded that primigravids were a high-risk factor for both maternal and perinatal outcome because they were prone to prolong second-stage labour and fetal distress.

The top three most common causes of CP in this study were birth asphyxia, CNS infections and kernicterus. This finding is in agreement with previous studies in Nigeria. ${ }^{[1,2,22]}$ In well-resourced environments, prenatal events have been reported to account for about $75 \%-95 \%$ of all cases of CP seen, ${ }^{[26,27]}$ while perinatal asphyxia only accounts for $6 \%-7 \% \cdot{ }^{[26]}$ Prenatal aetiologies include vascular injuries, placental conditions, maternal infections and genetic factors, amongst others. ${ }^{[26-28]}$ Vascular injuries such as periventricular haemorrhages could occur during the critical stages of brain development, particularly from the 24th to the 
Table 3. Pattern of motor type, topography, age and number of associated problems according to level of severity $(N=187)$

\begin{tabular}{|c|c|c|c|c|c|}
\hline \multirow[b]{2}{*}{ Variables } & \multicolumn{5}{|c|}{ GMFCS, $n(\%)$} \\
\hline & Level I & Level II & Level III & Level IV & Level V \\
\hline \multicolumn{6}{|l|}{ Motor type } \\
\hline Spastic $(n=136)$ & $13(9.6)$ & $17(12.5)$ & $6(4.4)$ & $21(15.4)$ & $79(58.1)$ \\
\hline Dyskinetic $(n=11)$ & $3(27.3)$ & $2(18.2)$ & 0 & $4(36.4)$ & $2(18.2)$ \\
\hline Ataxic $(n=17)$ & $4(23.5)$ & $13(76.5)$ & 0 & 0 & 0 \\
\hline Mixed $(n=19)$ & $2(10.5)$ & $7(36.8)$ & $4(21.1)$ & $4(21.1)$ & $2(10.5)$ \\
\hline Hypotonic $(n=4)$ & $0(0)$ & $1(25)$ & $1(25)$ & $1(25)$ & $1(25)$ \\
\hline \multicolumn{6}{|c|}{ Topographical distribution } \\
\hline Diplegia $(n=15)$ & 0 & $5(33.3)$ & $7(46.7)$ & $2(13.3)$ & $1(6.7)$ \\
\hline Hemiplegia $(n=42)$ & $15(35.7)$ & $17(40.5)$ & $2(4.8)$ & $6(14.3)$ & $2(4.8)$ \\
\hline Quadriplegia $(n=130)$ & $7(5.40)$ & $18(13.8)$ & $2(1.5)$ & $22(16.9)$ & $81(62.3)$ \\
\hline \multicolumn{6}{|c|}{ Age distribution of children (years) } \\
\hline $1-2(n=48)$ & $1(2.1)$ & $10(20.8)$ & $6(12.5)$ & $10(20.8)$ & $21(43.8)$ \\
\hline $2-4(n=44)$ & $2(4.5)$ & $10(22.7)$ & $4(9.1)$ & $10(22.7)$ & $18(40.9)$ \\
\hline $4-6(n=52)$ & $5(9.6)$ & $11(21.2)$ & $1(1.9)$ & $6(11.5)$ & $29(55.8)$ \\
\hline $6-12(n=43)$ & $14(32.6)$ & $9(20.9)$ & 0 & $4(9.3)$ & $16(37.2)$ \\
\hline \multicolumn{6}{|c|}{ Number of associated problems per child } \\
\hline None $(n=44)$ & $10(22.7)$ & $17(38.6)$ & $6(13.6)$ & $5(11.4)$ & $6(13.6)$ \\
\hline $1(n=70)$ & $8(11.4)$ & $16(22.9)$ & $4(5.7)$ & $14(20)$ & $28(40)$ \\
\hline $2(n=50)$ & $3(6)$ & $7(14)$ & $1(2)$ & $7(14)$ & $32(64)$ \\
\hline $3(n=15)$ & $1(6.7)$ & 0 & 0 & $4(26.7)$ & $10(66.7)$ \\
\hline$\geq 4(n=8)$ & 0 & 0 & 0 & 0 & $8(100)$ \\
\hline Total $(N=187)$ & 22 & 40 & 11 & 30 & 84 \\
\hline
\end{tabular}

34th week of gestation. For these reasons, the term hypoxic ischemic encephalopathy has been replaced with the term neonatal encephalopathy since, in most child deliveries, no evidence exists of either an acute hypoxia or ischaemic birth. Furthermore, only $13 \%$ of neonatal encephalopathy seen in newborn babies actually results in $\mathrm{CP}^{[23]}$ However, birth asphyxia remains a common cause of $\mathrm{CP}$ in developing nations such as Nigeria. Reasons for this include poor health service delivery, particularly in primary health centres, insufficient number of professional health workers, and lack of basic obstetric and neonatal resuscitation equipment. ${ }^{[9]}$

Most parents/caregivers of children with CP came from a lower socioeconomic background. Findings from this study are consistent with those of previous studies. ${ }^{[2,29]}$ Having a lower educational status does not leave many opportunities for a high-paying job. However, it was observed from this study that only $8 \%$ of parents/caregivers stopped at primary school education while over $50 \%$ went ahead to complete tertiary education. Nevertheless, the income of 118 (63.1\%) parents/caregivers was $<\$ 1000$ per month, which is equivalent to USD137 and about 3 days' minimum wage. ${ }^{[30]}$ This may imply that less purchasing power rather than ignorance resulted in mothers seeking poor antenatal and delivery care centres. ${ }^{[29]}$

$\mathrm{CP}$ can be classified either by using the nature of movement disorder (motor type) or topographical distribution. ${ }^{[26]}$ In this study, the most predominant movement disorder type was spastic $\mathrm{CP}$ (73.3\%). These findings were similar to previous studies done by Frank-Briggs and Alikor, ${ }^{[2]}$ and Ogunlesi et al. ${ }^{[29]}$ Four children $(\geq 4$ years at the time of this study) with CP (2.1\%) were observed to be hypotonic. Hypotonia is common during infancy; however, most children with CP will begin to develop spasticity, athetosis or ataxia transiently over the first two years of life. ${ }^{[31]}$ Permanent hypotonia has previously been described in children with $\mathrm{CP}$, most commonly associated with congenital $\mathrm{CP}^{[26]}$
Quadriplegia (67.4\%) was the most prevalent topographical distribution of $\mathrm{CP}$, in agreement with previous studies. ${ }^{[21,29]}$ About two-thirds $(67.6 \%)$ of children with spastic CP were quadriplegic. Both the spastic motor and quadriplegic sub-types of CP showed a high affinity for Level V of the GMFCS: $58.1 \%$ and $62.3 \%$ respectively. The GMFCS is an assessment tool used to determine both the severity of CP and likely prognosis. ${ }^{[15]}$ A child with a GMFCS of level V simply implies that voluntary control of movement is greatly restricted due to the child's body impairment and will require a high level of assistance from caregivers (parents, relatives or guardians) for mobility, usually requiring a wheelchair and a high level of dependence for other activities of daily living. ${ }^{[15]}$

There was a greater proportion of children with severe disability (GMFCS IV and V; 60.9\%) in this study, with only $33.2 \%$ of children presenting with mild CP (GMFCS I and II). This finding is contrary to the work done by Obembe et al., ${ }^{[32]}$ who reported that the prevalence of severe and mild disability was similar at $28.6 \%$ and $36.3 \%$ respectively. The results of this study may present a selection bias, as there is an active community awareness programme in Osun State, with education about what $\mathrm{CP}$ is and the role of physiotherapy in managing a child with CP. Mothers of children with severe disability are encouraged to bring their wards to the physiotherapy clinics for assessment and rehabilitation. This may explain the disparity observed between studies.

Children with mild disability (GMFCS I and II) tend to have fewer associated problems, while those with severe disability (GMFCS IV and V) tend to present with one or more associated problems. Associated problems are additional health conditions that are seen in children with $\mathrm{CP}$ which may affect the quality of life of the child. ${ }^{[3]}$ Associated problems include intellectual disability, seizure disorders, sleep disorder, pain, bladder incontinence and deafness. ${ }^{[3]}$ 
Table 4: Breakdown of the number of associated problems

\begin{tabular}{lc}
\hline Associated problems & $\boldsymbol{n}(\%)$ \\
\hline None & $44(23.5)$ \\
Speech impairment only & $48(25.7)$ \\
Learning disability only & $10(5.3)$ \\
Seizure disorders only & $11(5.9)$ \\
Oral motor impairment only & $1(0.5)$ \\
Speech impairment + seizure disorders & $15(8.0)$ \\
Speech impairment + intellectual disability & $6(3.2)$ \\
Speech impairment + oral motor impairment & $13(7.0)$ \\
Speech impairment + visual impairment & $2(1.1)$ \\
SI + BBCP & $14(7.5)$ \\
HI + VI + SI & $1(0.5)$ \\
SI + VI + SZD & $7(3.7)$ \\
SI + OMI + BBCP & $3(1.6)$ \\
SI + BBCP + EBP & $4(2.1)$ \\
HI + OMI + SZD + ID & $3(1.6)$ \\
VI + SZD + SI + OMI + BBCP & $1(0.5)$ \\
SI + SZD + BBCP + OMI & $4(2.1)$ \\
SI = speech impairment; BBCP = bladder and bowel control problems; \\
HI = hearing impairment; VI = visual impairment; SZD = seizure disorders; \\
OMI = oral motor impairment (including problems with feeding, swallowing \\
and drooling); EBP = emotional and behavioural problems; ID = intellectual \\
disability.
\end{tabular}

The GMFM is an assessment tool used by physiotherapist and other healthcare professionals to objectively determine the gross motor capacity of a child with CP. This observational instrument is usually used in clinics as an outcome measure to record changes in gross functional abilities of children with CP from baseline to a predetermined duration of physiotherapy or other therapeutic interventions. ${ }^{[16,17]}$ In this study, it was observed that the lower the gross motor functional scores (GMFM), the higher the level of severity (GMFCS Levels IV and V); while the higher the gross motor functional scores, the lower the level of severity (Levels I and II).

\section{Study limitations}

This study was a hospital-based (tertiary institutions only) study and so there is a possibility that our observations may not be a complete representation of all children with CP in Osun State. Also, data of respondents without cerebral palsy during this study were not collected. Therefore, a statistical analysis of the difference between children with $\mathrm{CP}$ and those without $\mathrm{CP}$ could not be computed. However, the pattern of children with CP seen in tertiary hospitals in Osun State has been highlighted.

\section{Conclusion}

Children with CP have various challenges with activities and participation as a result of impairments to their body structure. Management of children with CP by a paediatric physiotherapist is long-term and demanding. Developing locally relevant, standardised protocols/guidelines for the treatment of children with $\mathrm{CP}$ may

Table 5. Pattern of GMFM of children with cerebral palsy according to level of severity as determined by GMFCS

\begin{tabular}{|c|c|c|c|c|c|c|c|c|}
\hline \multirow[b]{2}{*}{ Variables } & & \multicolumn{7}{|c|}{ GMFCS, $n(\%)$} \\
\hline & & Level I & Level II & Level III & Level IV & Level V & $\chi^{2}$ & $p$-value \\
\hline \multicolumn{9}{|l|}{ GMFM } \\
\hline $0-20.9$ & 49 & $0(0)$ & $0(0)$ & $0(0)$ & $2(4.1)$ & 47 (95.9) & 291.82 & 0.002 \\
\hline $21-40.9$ & 51 & $0(0)$ & $0(0)$ & $0(0)$ & $17(33.3)$ & $34(66.7)$ & & \\
\hline $41-60.9$ & 31 & $0(0)$ & $8(25.8)$ & $10(32.3)$ & $10(32.3)$ & $3(9.7)$ & & \\
\hline $61-80.9$ & 21 & $1(4.8)$ & $18(85.7)$ & $1(4.8)$ & $1(4.8)$ & $0(0)$ & & \\
\hline $81-100$ & 35 & $21(60)$ & $14(40)$ & $0(0)$ & $0(0)$ & $0(0)$ & & \\
\hline Total & 187 & 22 & 40 & 11 & 30 & 84 & & \\
\hline
\end{tabular}

Table 6. Association between each of the level of severity, socioeconomic status, topography, motor type, associated problems and birth order

\begin{tabular}{|c|c|c|c|c|c|c|c|}
\hline Variables & & GMFCS & SES & Topography & Motor type & AP & Birth order \\
\hline \multirow[t]{2}{*}{ GMFCS } & $\chi^{2}$ & & & & & & \\
\hline & $p$ & 1.000 & & & & & \\
\hline \multirow[t]{2}{*}{ SES } & $x^{2}$ & 22.42 & & & & & \\
\hline & $p$ & $0.004^{\star}$ & 1.000 & & & & \\
\hline \multirow[t]{2}{*}{ Topography } & $x^{2}$ & 144.26 & 11.76 & & & & \\
\hline & $p$ & $0.001^{*}$ & 0.162 & 1.000 & & & \\
\hline \multirow[t]{2}{*}{ Motor type } & $\chi^{2}$ & 74.79 & 27.62 & 55.55 & & & \\
\hline & $p$ & $0.001^{*}$ & $0.001^{*}$ & $0.001^{*}$ & 1.000 & & \\
\hline \multirow[t]{2}{*}{ AP } & $\chi^{2}$ & 53.22 & 14.70 & 64.67 & 11.62 & & \\
\hline & $p$ & $0.001^{*}$ & 0.065 & $0.001^{*}$ & 0.770 & 1.000 & \\
\hline \multirow[t]{2}{*}{ Birth order } & $x^{2}$ & 55.77 & 29.05 & 61.04 & 26.71 & 43.65 & 1.000 \\
\hline & $p$ & 0.001 & 0.001 & 0.001 & 0.144 & 0.002 & 1.000 \\
\hline
\end{tabular}


optimise the physiotherapy management of these children, and improve functional outcomes. This study provides information regarding the pattern of $\mathrm{CP}$ in children in Osun State, Nigeria, as one of the first steps to develop a physiotherapy management protocol for the region.

Acknowledgements. The authors gratefully acknowledge the parents of the study respondents for giving consent to participate in the study. Author's contribution. JOO: conceptualised the study, collected and analysed the data and wrote the manuscript. SAA: analysis and critical review of the manuscript. KOO: analysis and critical review of the manuscript. OAA: critical review of the manuscript. All the authors approved the final version of the manuscript.

\section{Funding. None.}

Conflicts of interest. None.

1. Lagunju IA, Okafor, OO. An analysis of disorders seen at the paediatric neurology clinic, University College Hospital, Ibadan, Nigeria. West Afr J Med 2009;28(1):38-42. https://doi.org/10.4314/wajm.v28i1.48424

2. Franks-Briggs AI, Alikor EAD. Sociocultural issues and causes of cerebral palsy in Port Harcourt, Nigeria. Nig J Paediatr 2011;38(3):115-119. https://doi. org/10.4314/njp.v38i3.72266

3. Novak I, Hines M, Goldsmith S, Barclay R. Clinical prognostic messages from a systematic review on Cerebral Palsy. Pediatr 2012;130(5):e1285-1312. https:// doi.org/10.1542/peds.2012-0924

4. Reddihough DS, Collins KJ. The epidemiology and causes of cerebral palsy. Aus J Physiother 2003;49(1):7-12. https://doi.org/10.1016/S0004-9514 (14)60183-5

5. Olney SJ, Wright MJ. Cerebral palsy. In: Campbell SK, Vander-Linden DW, Palisano RJ, eds. Physical Therapy for Children. St Louis: Saunders-Elsevier. 2006:625-664.

6. Dambi JM, Jelsma J, Mlambo T. Caring for a child with cerebral palsy: The experience of Zimbabwean mothers. Afri J Disabil 2015;4(1):168. https://doi. org/10.4102/ajod.v4i1.168

7. El-Tallawy HN, Farghaly WMA, Shehata GA, et al. Cerebral palsy in Al-Quseir City, Egypt: Prevalence, subtypes, and aetiology. Neuropsychiatr Dis Treat 2014;10:1267-1272. https://doi.org/10.2147/NDT.S59599

8. Couper J. Prevalence of childhood disability in rural KwaZulu-Natal. S Afr Med J 2002;92(7):549-552. https://www.ajol.info/index.php/samj/article/ viewFile/132073/121672

9. Duru EJ, Nwagbos CI. The problems and prospects of public health care development in Nigeria's local government system. Glob J Soc Sci 2007;6(1):5156. https://doi.org/10.4314/gjss.v6i1.22826

10. Alikor EAD. Common Growth and Development Problems. In: Azubuike JC, Nkanginieme KEO, eds. Paediatrics and Child Health in a Tropical Region. Port Harcourt: University of Port Harcourt Press, 2007:70-83.

11. Potharaju NR. Seizures in Cerebral Palsy. Indian J Cereb Palsy 2016;2(1):3-21. https://doi.org/10.4103/2395-4264.188150

12. Johnston MV. Encephalopathies. In: Kliegman RM, Behrman RE, Jenson HB, Stanton BF, eds. Nelson Textbook of Pediatrics. 18th ed. Philadelpha: SaundersElsevier; 2007:680

13. Anttila H, Autti-Rämö I, Suoranta J, Mäkelä M, Malmivaara A. Effectiveness of physical therapy interventions for children with cerebral palsy: A systematic review. BMC Pediatr 2008;8:14. https://doi.org/10.1186/1471-2431-8-14
14. Patel DR. Therapeutic interventions in cerebral palsy. Indian J Pediatr 2005; 72(11):979-983. https://doi.org/10.1007/bf02731676

15. Palisano RJ, Rosenbaum P, Bartlett D, Livingston MH. Content validity of the expanded and revised Gross Motor Function Classification System. Dey Med Child Neurol 2008;50(10):744-750. https://doi.org/10.1111/j.1469 8749.2008.03089.x

16. Palisano RJ, Hanna SE, Rosenbaum PL, et al. Validation of a model of gross motor function for children with cerebral palsy. Phys Ther 2000;80(10):974-985. https:// doi.org/10.1093/ptj/80.10.974

17. Alotaibi M, Long T, Kennedy E, Bavishi S. The efficacy of GMFM- 88 and GMFM 66 to detect changes in gross motor function in children with cerebral palsy $(\mathrm{CP})$ A literature review. Disabil Rehabil 2014;36(8):617-627. https://doi.org/10.3109/ 09638288.2013.805820

18. Ogunlesi TA, Dedeke IOF, Kuponiyi OT. Socio-economic classification of children attending specialist paediatric centres in Ogun State, Nigeria. Nig Med Practitioner 2008;54(1):21-25. https://doi.org/10.4314/nmp.v54i1.28943

19. Oyedeji GA. Socioeconomic and cultural background of hospitalized children in Ilesha. Nig J Paediatr 1985;12(4):111-117.

20. Peters GO, Adetola A, Fatudimu MB. Review of paediatric neurological conditions seen in the physiotherapy department of a children's hospital in Ibadan, Nigeria. Afr J Biomed Res 2008;11:281-284. https://doi.org/10.4314/ajbr.v11i3.50735

21. Hamzat TK, Fatudimu MB. Caregivers or care providers: Who should assess motor function in cerebral palsy? J Pediatr Neurol 2008;6(4):345-350. https://doi. org/10.1055/s-0035-1557480

22. Okike CO, Onyire BN, Ezeonu CT, Agumadu HU, Adeniran KA, Manyike PC Cerebral palsy among children seen in the neurology clinic of Federal Medical Centre (FMC), Asaba. J Community Health 2013;38(2):257-260. https://doi. org/10.1007/s10900-012-9608-2

23. MacLennan AH, Thompson SC, Gecz J. Cerebral palsy: Causes, pathways, and the role of genetic variants. Am J Obstet Gynecol 2015;213(6):779-788. https:// doi.org/10.1016/j.ajog.2015.05.034

24. Indra, Usharani N, Bendigeri M. A study on clinical outcome of obstructed labour. Int J Reprod Contracept Obstet Gynecol 2017;6(2):439-442. https://doi. org/10.18203/2320-1770.ijrcog20170027

25. Hashim N, Nagvi S, Khanam M, Jafry HF. Primiparity as an intrapartum obstetric risk factor. J Pak Med Assoc 2012;62(7):694-698

26. Beaman J, Kalisperis FR, Miller-Skomorucha K. The infant and child with cerebra palsy. In: Tecklin JS, ed. Pediatric Physical Therapy. Philadelphia: Lippincott Wolters Klumer; 2015:187-246.

27. Nelson KB, Blair E. Prenatal factors in singletons with cerebral palsy born at or near term. New Engl J Med 2015;373(10):946-953. https://doi.org/10.1056/ nejmra1505261

28. Neufeld MD, Frigon C, Graham AS, Mueller BA. Maternal infection and risk of cerebral palsy in term and preterm infants. J Perinatol 2005;25(2):108-113. https://doi.org/10.1038/sj.jp.7211219

29. Ogunlesi T, Ogundeyi M, Adekanmbi F, Fetuga B, Ogunfowora O, Olowu A Socio-clinical issues in cerebral palsy in Sagamu, Nigeria. S Afr J Child Health 2008;2(3):120-124.

30. United States Department of Labor. Wage and Hour Division (WHD): Minimum Wage Laws in the States - July 1, 2017. https://www.dol.gov/whd/minwage/ america.htm (accessed 1 September 2017).

31. Miller F. Cerebral palsy management. In: Physical Therapy of Cerebral Palsy. New York: Springer; 2007:51-106.

32. Obembe AO, Johnson OE, Olaogun MOB, Ogunleye MC. Gross motor function in cerebral palsy: The association with motor type and topographical distribution. Nig J Med Rehab 2013;16(2):1-17.

Accepted 1 February 2018. 